\title{
烯基溴化合物的合成方法研究进展
}

\author{
张文生 $*, a$ 许文静 $a$ 匡春香 $*, b$ \\ ( ${ }^{a}$ 焦作师范高等专科学校理工学院 焦作 454001) \\ ( ${ }^{b}$ 同济大学化学系 上海 200092)
}

\begin{abstract}
摘要 以烯基澳化合物为代表的烯基卤代烃(1-卤代-1-烯烃)是过渡金属催化下通过 $\mathrm{C}-\mathrm{C}$ 偶联反应构建多取代烯烃的 重要合成砌块. 因此, 开发简便、有效的合成烯基澳化合物的新方法越来越受到人们的重视和关注. 对按照所使用的合 成原料进行分类，对近年来所报道的烯基澳化合物的合成方法进行了回顾. 所综述的主要的合成方法中所涉及的合成 原料涵盖了酮类、炔类、炔丙醇类、烯基三氟甲磺酸酯类、3-芳基-2,3-二溴丙酸、芐基溴类、丙烯酸类、环丙烯类和 烯丙醇类衍生物等共 9 类化合物.
\end{abstract}

关键词＼cjkstart烯基溴化合物; 合成; 立体选择性; 区域选择性

\section{Research Advances in the Synthetic Methods of Vinyl Bromides}

\author{
Zhang, Wensheng ${ }^{*, a} \quad \mathrm{Xu}$, Wenjing $^{a} \quad$ Kuang, Chunxiang ${ }^{*, b}$ \\ $\left({ }^{a}\right.$ School of Technology, Jiaozuo Teachers College, Jiaozuo 454001) \\ ( ${ }^{b}$ Department of Chemistry, Tongji University, Shanghai 200092)
}

\begin{abstract}
Vinyl halides (1-halo-1-alkenes), especially vinyl bromides, represent a kind of organic synthetic blocks, which play important roles in the construction of polysubstituted alkenes through transition metal-catalyzed $\mathrm{C}-\mathrm{C}$ cross-coupling reactions. Consequently, there have been much attentions in facile, efficient methods of preparing vinyl bromides. According to the types of the synthetic substrates used in the literatures, newly reported means on the synthesis of vinyl bromides in recent years are surveyed. The synthetic substrates involved in these new approaches include ketones, alkynes, propargylic alcohols, vinyl triflates, 3-aryl-2,3-dibromopropionic acids, benzyl bromides, acrylic acids, cyclopropenes and allylic alcohol derivatives, etc.

Keywords vinyl bromide; synthesis; stereoselectivity; regioselectivity
\end{abstract}

烯基卤代烃(1-卤代-1-烯烃)是过渡金属催化下通过 $\mathrm{C}-\mathrm{C}$ 偶联反应构建多取代烯烃的重要合成砌块 ${ }^{[1]}$. 此 外, 烯基卤代烃还可广泛应用于制备烯基锂 ${ }^{[2] 、}$ 烯基格 氏试剂和自由基反应底物 ${ }^{[3]}$ 以及合成 $\alpha$-卤代酩 ${ }^{[4]}$ 和杂环 化合物 ${ }^{[5]}$ 等. 从应用范围、制备成本和反应的立体和区 域选择性等方面综合来看, 烯基澳化合物(1-澳-1-烯烃, Scheme 1)都是最具有代表性的烯基卤代烃类型. 烯基 溴化合物的传统合成方法包括: 以醛为原料的 Takai 烯 化法 ${ }^{[6]}$ 、以肉桂酸衍生物为原料通过脱羧溴代的 Hunsdiecker 反应 ${ }^{[7]}$ 和以 1,1-二澳-1-烯为原料的选择性 还原法 ${ }^{[8]}$. 近年来, 随着金属催化有机偶联反应的发展,
研究和开发新的更为有效的合成烯基溴化合物的新方 法越来越引起人们的关注. 本文按照合成方法中所使用 的原料分类，对近年来烯基溴化合物的合成方法研究进 展进行综述.<smiles>[R]C([R])=C([Y])Br</smiles>

\section{Scheme 1}

\section{1 以酮为原料}

Prati 等 ${ }^{[9]}$ 以 $\mathrm{CH}_{2} \mathrm{Cl}_{2}$ 为溶剂, 以酮为原料(Eq. 1), 合

\footnotetext{
* E-mail: tongjizws@163.com

Received October 9, 2013; revised November 6, 2013; published online November 20, 2013.
}

Project supported by the National Natural Science Foundation of China (No. 21272174), the Foundation of He'nan Scientific and Technological Committee (Nos. 132300410206, 132300410167) and the Foundation of Jiaozuo Scientific and Technological Bureau (No. 2012017).

国家自然科学基金(No. 21272174)、河南省科技厅基础和前沿研究计划(Nos. 132300410206, 132300410167)、焦作市科技局科技计划(No. 2012017)资 助项目. 
成了相应的烯基溴化合物. 在 $\mathrm{Et}_{3} \mathrm{~N}$ 作用下，酮和 $(\mathrm{PhO})_{3} \mathrm{PBr}_{2}$ 在 $-60{ }^{\circ} \mathrm{C}$ 下低温反应 $18 \mathrm{~h}$, 恢复至室温后 再回流反应 $2 \mathrm{~h}$, 即可高收率地转化为相应的烯基溴化 合物.

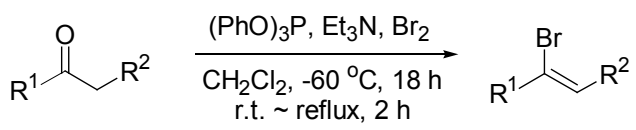

$R^{1}, R^{2}=$ aryl or alkyl

\section{2 以炔为原料}

\section{1 以炔和 NBS 为原料}

Hoveyda 等 ${ }^{[10]}$ 以端基炔 (Scheme 2) 为原料, 在 $\mathrm{Ni}(\mathrm{dppp}) \mathrm{Cl}_{2}$ 催化下, 首先和 $\mathrm{HAl}(\mathrm{Bu}-i)_{2}$ 进行加成反应, 室温条件下转化为 $\alpha$-烯基铝化合物，反应的区域选择性 超过了 95\%. 该烯基铝化合物中间体和 $N$-溴代丁二酰 亚胺(NBS)在室温下反应 $1 \mathrm{~h}$ 即可转化为相应的 $\alpha$-烯基 溴化合物, $\alpha$-区域选择性高于 $98 \%$.

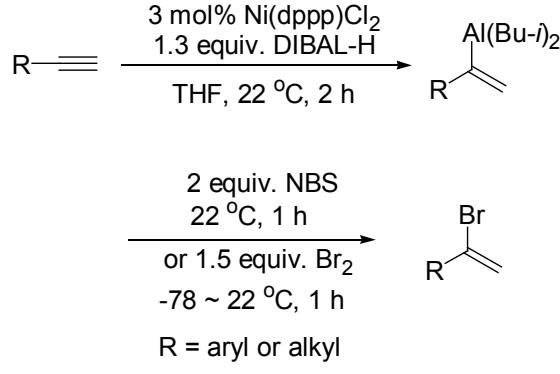

Scheme 2

\section{2 以炔、乙酸酐和 $N$-溴代丁二酰亚胺为原料}

Jiang 课题组 ${ }^{[11]}$ 以端基炔、乙酸䣶和 NBS 为原料(Eq. 2), 在 $\mathrm{AgBF}_{4}$ 催化下, 通过三组分反应, 高区域选择性 和立体选择性地合成了有价值的( $Z$ - $-\beta$-溴代乙烯醇的乙 酸酯. 端基炔首先和 $\mathrm{Ag}^{+}$结合生成 $\mathrm{Ag}$ 盐, 再和 NBS 反 应生成了端基炔的澳化物中间体. 炔的溴化物在 $\mathrm{Ag}^{+}$的 作用下，接受乙酰基的亲电进攻生成相应的双功能化产 物.

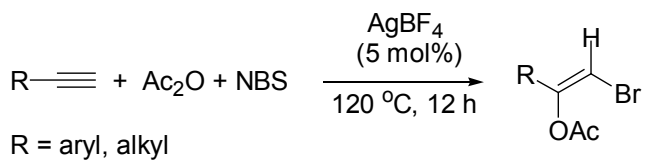

\section{3 以炔和仲醇为原料}

Kabalka 等 ${ }^{[12]}$ 研究发现, 仲醇的锂盐在 $\mathrm{TiBr}_{4}$ 的诱导 下, 可以和炔之间发生偶联, 得到三取代的(E)-烯基溴 化合物(Eq, 3).

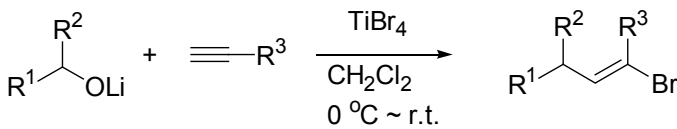

$$
\begin{aligned}
& \mathrm{R}^{1}=\text { aryl, alkynyl; } \mathrm{R}^{2}=\mathrm{Ph}, 4-\mathrm{ClC}_{6} \mathrm{H}_{4}, 4-\mathrm{MeOC}_{6} \mathrm{H}_{4} \\
& \mathrm{R}^{3}=\mathrm{Ph}, 4-\mathrm{MeC}_{6} \mathrm{H}_{4}
\end{aligned}
$$

\section{3 以芳基乙烯为原料}

具有 $(E)$-或 $(Z)$-立体选择性的 $\beta$-溴代芳乙烯是一类 双键和苯环共轭特殊结构的烯基溴化合物. 该类化合物 可通过偶联反应构建芳基烯烃化合物. 本课题组已对 2007 年前的主要合成方法进行了综述 ${ }^{[13]}$. Pawluć 等 ${ }^{[14]}$ 报道了一种以芳基乙烯为原料立体选择性合成 $(E)-\beta$-溴 代芳乙烯的方法(Eq. 4). 该方法通过 Ru-催化下的硅基 化偶联反应和 NBS 为溴代试剂的脱硅溴代反应，“一锅 法”两步反应将芳基乙烯转化为 $(E)-\beta$-溴代芳乙烯. 第一 步反应以甲苯为溶剂, 只需催化量的 $\mathrm{RuH}(\mathrm{Cl})$ $(\mathrm{CO})\left(\mathrm{PPh}_{3}\right)_{3}$ 催化剂. 第二步的反应过程中加入 5 倍于甲 苯体积的乙腈, 对脱硅澳代过程至关重要. 苯环上存在 的供电子和吸电子基团对于反应的高产率( $89 \%$ ～94\%) 和高的 $(E)$-立体选择性 $(>98 \%)$ 几乎没有影响.

$$
\begin{aligned}
& \text { (1) } \mathrm{CH}_{2}=\mathrm{CHSiMe}_{3} \text { (1 equiv.) } \\
& \mathrm{RuH}(\mathrm{Cl})(\mathrm{CO})\left(\mathrm{PPh}_{3}\right)_{3}(1 \mathrm{~mol} \%) \\
& \mathrm{Ar} \curvearrowright \frac{\text { toluene, } 100^{\circ} \mathrm{C}, 6 \mathrm{~h}}{\text { (2) NBS, MeCN, r.t., } 1 \mathrm{~h}} \mathrm{Ar} \curvearrowright \mathrm{Br}
\end{aligned}
$$

\section{4 以炔丙醇及其衍生物为原料}

\section{1 以炔丙醇的乙酸酯为原料合成 $\alpha$-溴代烯酮}

$\alpha$-澳代烯酮是一种重要的合成中间体. Zhang 等 ${ }^{[15]}$ 报道了以炔丙基醇乙酸酯为原料, NBS 为 $\mathrm{Br}^{+}$源，在 $\mathrm{Au}\left(\mathrm{PPh}_{3}\right) \mathrm{NTf}_{2}$ 催化下合成 $\alpha$-溴代烯酮的方法(Eq. 5). 所 使用的炔丙基乙酸酯可以醛(酮)为原料简便地制备. 该 合成方法中，溶剂的选择很关键，丙酮是最理想的溶剂. 而反应体系中加入的微量水，对产物的立体选择性至关 重要.

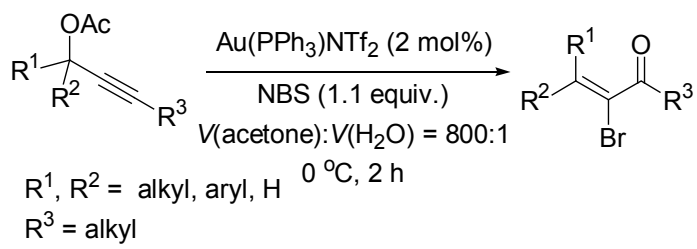

\section{2 直接以炔丙醇为原料合成 $\alpha$-溴代烯酮}

由于炔丙基乙酸酯需要以炔丙基醇为原料制备, Zhang 等 ${ }^{[16]}$ 进一步探讨了直接以炔丙基醇为原料合成 $\alpha$ 溴代烯酮的可能性. 当以炔丙基醇为原料时，需要用 
$\mathrm{Ph}_{3} \mathrm{PAuNTf}_{2}$ 和 $\mathrm{MoO}_{2}(\mathrm{acac})_{2}$ 两种金属试剂共催化, 同时 加入 $\mathrm{Ph}_{3} \mathrm{PO}$ 以抑制烯酮(醛)副产物的生成. 该反应以 $\mathrm{CH}_{2} \mathrm{Cl}_{2}$ 为溶剂, 在室温下可以顺利完成(Eq. 6).

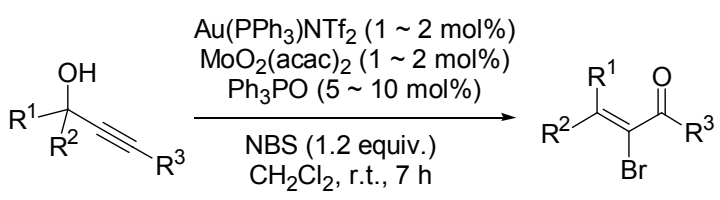

$R^{1}, R^{2}, R^{3}:$ alkyl, aryl, $H$

\section{5 以三氟甲磺酸酯为原料}

\section{$5.1 \mathrm{Pd}$-催化下以三氟甲磺酸酯为原料}

Buchwald 等 ${ }^{[17]}$ 报道了第一例将芳基或烯基三氟甲 磺酸酯转化为相应的芳基(烯基)溴化物的方法(Eq. 7). 该反应在 $\mathrm{Pd}$ 催化下完成. 由于 $\mathrm{KBr}$ 在反应体系中溶解 性较差, 需加入相转移催化剂 PEG3400 增加其溶解性. 反应体系中还需加入 1.5 equiv. 的三异丁基铝作为 Lewis 酸, 可以消耗掉转化过程中释放出来的副产物 KOTf, 明显提高反应转化率. 同时, 加入 1.5 equiv. 的 2-丁酮作 为助剂, 对于抑制偶联和还原副反应的发生和保证反应 顺利完成至关重要. 但是, 该方法主要适用于以三氟甲 磺酸酯为底物来合成澳代芳烃. 所报道的 2 例合成的烯 基溴产物中, 均是由环状的烯基三氟甲磺酸酯合成环状 烯基产物.

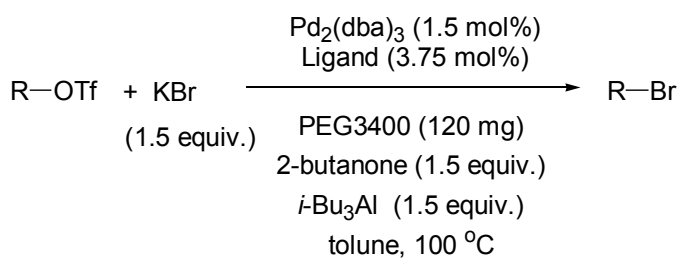

R: aryl, vinyl

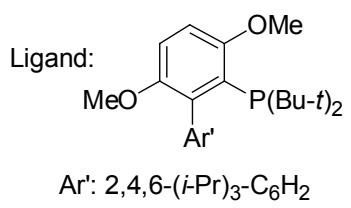

为了避免大量使用各种反应添加物和相转移催化 剂, Buchwald 等 ${ }^{[18]}$ 对上述方法进行了进一步的改进. 他 们选择 dixoane 为反应溶剂, 增加 $\mathrm{KBr}$ 的用量至 2 equiv., 加入 0.5 equiv. 的 KF 作为唯一的反应添加物, 就可以顺 利地将芳基或烯基三氟甲磺酸酯转化为相应的芳基(烯 基)溴化物(Eq. 8). 该报道共涉及 29 例的合成实例，其 中有 5 例为环状的烯基澳产物.

\section{$5.2 \mathrm{Ru}$-催化下以三氟甲磺酸酯为原料}

Shirakawa 和 Hayashi 课题组 ${ }^{[19]}$ 报道了 $\mathrm{Ru}$ 催化下

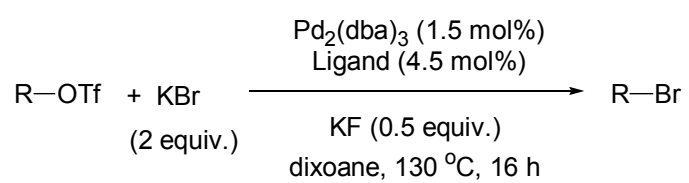

$$
\text { R: aryl, vinyl Ligand: }
$$

芳基或烯基三氟甲磺酸酯转化为相应的芳基(烯基)溴化 物的方法. 在 $\left[\mathrm{Cp} * \mathrm{Ru}(\mathrm{MeCN})_{3}\right] \mathrm{OTf}$ 催化下, 芳基磺酸酯 为底物的反应最佳温度是 $100{ }^{\circ} \mathrm{C}$, 而五元或六元的环 状烯基三氟甲磺酸酯则可以在室温 $\left(25{ }^{\circ} \mathrm{C}\right)$ 下 顺利转化 为相应的烯基溴产物(Eq. 9).

$$
\text { OOTf }+ \text { LiBr } \frac{\substack{\mathrm{DMl}, 2{ }^{\circ} \mathrm{C} \\ 10 \mathrm{~min} \sim 2 \mathrm{~h}}}{(5 \mathrm{~mol} \%)}
$$

该课题组还发现，非环状的烯醇三氟甲磺酸酯在同 样的条件下, 也可高产率地完成转化. 但是在 $25{ }^{\circ} \mathrm{C}$ 下 反应，产物的构型会发生异构化. 长时间放置(7 d) 后产 物的 $E / Z$ 比例逐步稳定到 36/64. 而控制反应温度为一 $20{ }^{\circ} \mathrm{C}$ 时, 即使以 $E / Z$ 两种构型的烯基三氟甲磺酸酯混 合物为原料, 也可以得到高立体选择性的 $(E)$-烯基澳化 物(Eq. 10). 因而, 该 Ru 催化下的反应提供了一种合成 高立体选择性开链 $(E)$-烯基溴的重要方法.

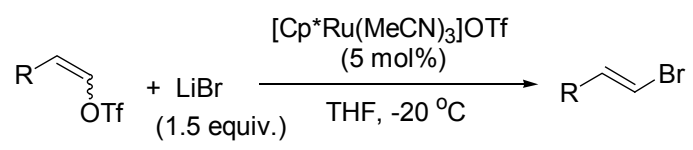

\section{6 以肉桂酸二溴加成物和 $\mathrm{NaN}_{3}$ 为原料}

Kuang 课题组 ${ }^{[20]}$ 在对反式-3-(4-氯磺酰苯基)-2,3-二 溴丙酸进行叠氮化反应研究时发现, 加入 2 equiv.的 $\mathrm{NaN}_{3}$, 不仅可以完成磺酰氯的叠氮化反应, 还可以同时 完成 2,3-二澳丙酸的脱羧脱溴(Eq. 11)反应，高立体选择 性的得到(Z)- $\beta$-芳基溴乙烯产物. 过量 1 倍的 $\mathrm{NaN}_{3}$ 代替 了传统的 2,3-二澳丙酸脱羧脱澳反应中所需要的 $\mathrm{Et}_{3} \mathrm{~N}^{[21]}$.

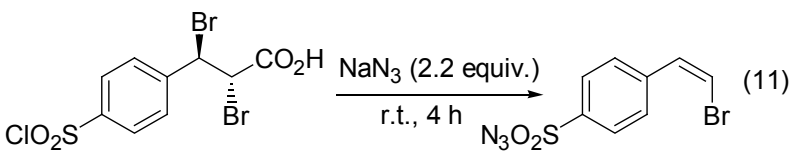

\section{7 以苄基溴为原料}

Charette 等 ${ }^{[22]}$ 报道, 芐基溴衍生物在 $-78{ }^{\circ} \mathrm{C}$ 低温 下和过量(4 equiv.)的 $\mathrm{CH}_{2} \mathrm{Br}_{2}$ 反应, 可以转化为相应的 
1,1-二溴苯甲烷衍生物中间体。该中间体在过量 (3 equiv.)的碱 NaHMDS 作用下消除 $\mathrm{HBr}$ 分子, 可以得到高 立体选择性的 $(E)-\beta$-芳基烯基溴化合物, $(E)$-构型的产物 比例高于 $99 \%$. 该反应条件对苯环上的供电子基团 ( $\mathrm{MeO}$ 等)和吸电子基团( $\mathrm{F}$ 等)以及卤素基团 $(\mathrm{Br})$ 都有很 好的兼容性(Eq. 12).

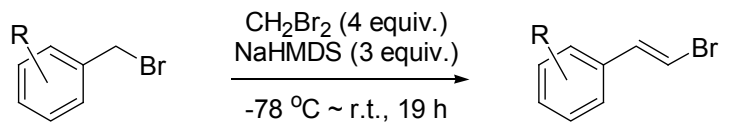

$\mathrm{R}: \mathrm{H}, 4-\mathrm{Me}, 4-\mathrm{MeO}, 4-\mathrm{F}, 2-\mathrm{Br}$

\section{8 以丙烯酸及其相应衍生物为原料}

$\mathrm{Li}$ 等 ${ }^{[23]}$ 报道, 以丙烯酸及其酯(腈、醛或相应结构的 酮)为原料, 和溴进行简单加成后得到 $\alpha, \beta$-二溴代丙酸 酯(腈、醛或酥)等 Michael 反应受体. 该类中间体在 DMSO 中加热至 $60 \sim 80{ }^{\circ} \mathrm{C}$, 可以高效且立体选择性的 脱去 $\beta$-溴, 得到 $\alpha$-溴代丙烯酸酯衍生物(Scheme 3 ). 在 $\alpha$-位引入溴后, 该 Michael 反应受体具有重要的合成价 值.

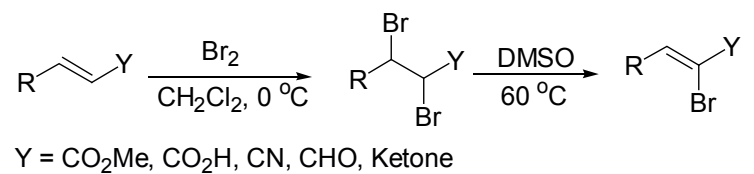

\section{Scheme 3}

\section{9 以环丙烯衍生物为原料}

Lam 等 ${ }^{[24]}$ 以双活化的取代环丙烯和等量的 $\mathrm{MgBr}_{2}$ 之间的高立体选择性的开环反应, 合成了一组多取代的 烯基溴化合物(Eq. 13). 该开环反应具有高度的区域和 立体选择性. 开环位置发生在取代基少的双键一侧, 开 环后的烯基溴产物则具有专一的 $(E)$-立体构型.

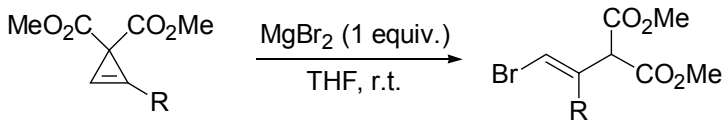

$\mathrm{R}=\mathrm{Ph}, \mathrm{CH}_{2} \mathrm{CH}_{2} \mathrm{Ph}, p-\mathrm{Tol}, \mathrm{CH}_{2} \mathrm{CH}_{2} \mathrm{OBz}, n-\mathrm{Bu}$

\section{0 以烯丙醇衍生物为原料}

\section{1 以 1 -芳基烯丙醇为原料合成 $\alpha$-溴代不饱和酮}

Chisholm 等 ${ }^{[25]}$ 报道, 使用过量的 Moffatt-Swern 试 剂( 3 equiv. 的 BrCOCOBr +4 equiv. 的 DMSO), 通过氧化 一双键加溴-消除的三步串联反应，可将芳基烯丙醇转化 为具有 $\alpha$-位区域选择性的溴代不饱和酮(Eq. 14).

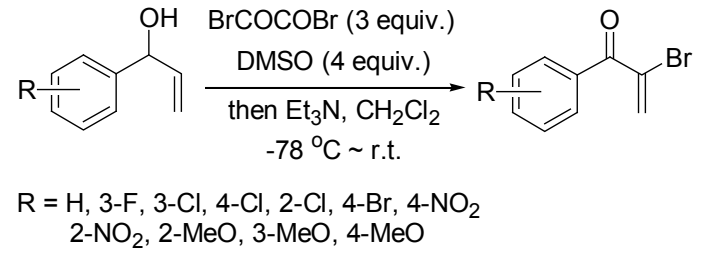

\section{2 以烯丙醇的醚为原料}

Kutsumura 和 Saito 等 ${ }^{[26]}$ 以烯丙基醇衍生物为原料, 通过加成溴化和 Ohgiya-Nishiyama 法选择性还原，“一 锅法” 合成了一组 2-溴烯丙基醇衍生物(Scheme 4). 他 们的研究发现, 以 pydidine $\cdot \mathrm{HBr}_{3}$ 为溴化试剂, 在室温下 完成双键的加溴反应后，再用 DBU (3.1 equiv.)选择性的 消除 1 号位上的溴，高区域选择性的合成 $\beta$-溴烯丙基醇 衍生物(方法 A). 第一步反应时加入 $\mathrm{K}_{2} \mathrm{CO}_{3}$ (1.1 equiv.), 则第二步反应 DBU 的用量可以降低至 1.1 equiv.

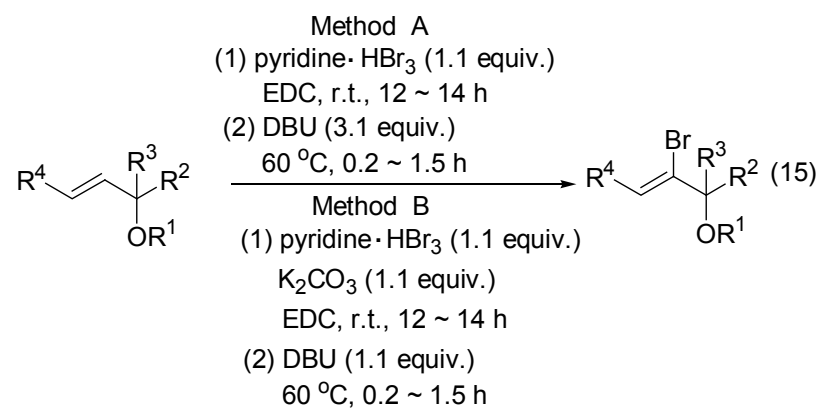

$$
\begin{aligned}
& \mathrm{R}^{1}=\text { aryl, benzyl, TIPS, COAr } \\
& \mathrm{R}^{2}, \mathrm{R}^{3}=\mathrm{H}, \mathrm{Me} \\
& \mathrm{R}^{4}=\mathrm{H}, \mathrm{Pr}-n
\end{aligned}
$$

更为重要的是，向方法 $\mathrm{B}$ 的反应体系中，加入端基 炔烃, 在 $\mathrm{CuI}-\mathrm{PdCl}_{2}\left(\mathrm{PPh}_{3}\right)_{2}$ 催化下, 可以直接完成 Sonogashira 偶联反应，通过 “一锅法” 三步在烯丙醇的 2 号位引入炔基，构建烯炔类化合物(Scheme 4).

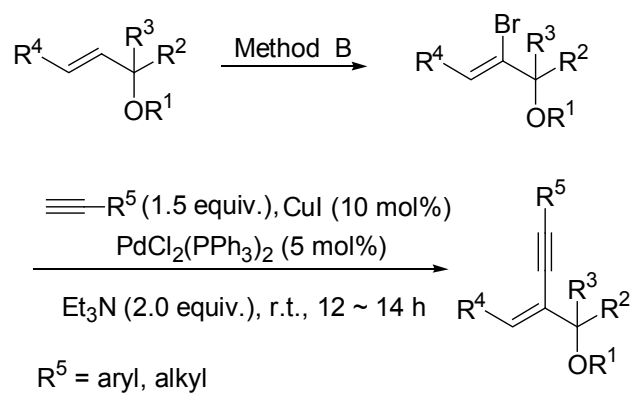

\section{Scheme 4}

\section{1 回顾与展望}

近年来，烯基溴化合物的合成原料出现了多元化的 趋势. 本文综述的合成方法中所使用的原料种类达到 9 种. 同时, 对烯基溴合成中的立体选择性 $(E$-或 $Z$-选择) 
和区域选择性 $(\alpha$-或 $\beta$-选择)要求也越来高. 此外, $\beta$-溴 代乙烯醇酯 ${ }^{[11]} 、 \alpha$-溴代烯酮/醛 ${ }^{[16]}$ 等具有特殊结构的双 官能团烯基溴化合物的合成方法也逐渐出现, 为金属催 化下的偶联反应提供了更加丰富的反应底物. 随着金属 有机化学的迅速发展, 研究和发现高效和低成本的烯基 溴化合物合成方法也变得越来越重要.

\section{References}

[1] Kutsumura, N.; Niwa, K.; Saito, T. Org. Lett. 2010, 12, 3316.

[2] (a) Poss, C. S.; Rychnovsky, S. D.; Schreiber, S. L. J. Am. Chem. Soc. 1993, 115, 3360 .

(b) Paqutte, L. A.; Hofferberth, J. E. J. Org. Chem. 2003, 68, 2266.

[3] Yokota, M.; Toyota, M.; Ihara, M. Chem. Commun. 2003, 422.

[4] (a) Vanbrunt, M. P.; Ambenge, R. O.; Weinreb, S. M. J. Org. Chem. 2003, 68, 3323.

(b) Novikov, Y. Y.; Sampson, P. J. Org. Chem. 2005, 70, 10247.

[5] (a) Ohno, H.; Yamamoto, M.; Iuchi, M.; Tanaka, T. Angew. Chem., Int. Ed. 2005, 44, 5103.

(b) Miyamoto, H.; Okawa, Y.; Nakazaki, A.; Kobayashi, S. Tetrahedron Lett. 2007, 48, 1805.

(c) Suzuki, H.; Sakai, N.; Iwahara, R.; Fujiwara, T.; Satoh, M.; Kakehi, A.; Konakahara, T. J. Org. Chem. 2007, 72, 5878.

[6] (a) Takai, K.; Nitta, K.; Utimoto, K. J. Am. Chem. Soc. 1986, 108, 7408 .

(b) Takai, K.; Ichiguchi, T.; Hikasa, S. Synlett 1999, 1268.

[7] (a) Naskar, D.; Roy, S. Tetrahedron 2000, 56, 1369.

(b) Das, J. P.; Roy, S. J. Org. Chem. 2002, 67, 7861.

(c) You, H.-W.; Lee, K.-J. Synlett 2001, 105.

[8] (a) Hirao, T.; Masunaga, T.; Ohshiro, Y.; Agawa, T. J. Org. Chem. 1981, 46, 3745 .

(b) Abbas, S.; Hayes, C. J.; Worden, S. Tetrahedron Lett. 2000, 41, 3215 .

(c) Kuang, C.; Senboku, H.; Tokuda, M. Tetrahedron 2002, 58,
1491.

(d) Horibe, H.; Kondo, K.; Okuno, H.; Aoyama, T. Synthesis 2004, 986.

[9] Spaggiari, A.; Vaccari, D.; Davoli, P.; Torre, G.; Prati, F. J. Org. Chem. 2007, 72, 2216.

[10] Gao, F.; Hoveyda, A. H. J. Am. Chem. Soc. 2010, 132, 10961.

[11] Chen, Z. W.; Li, J. H.; Jiang, H. F.; Zhu, S. F.; Li, Y. B.; Qi, C. R. Org. Lett. 2010, 12, 3262.

[12] Yao, M. L.; Quick, T. R.; Wu, Z. Z.; Quinn, M. P.; Kabalka, G. W. Org. Lett. 2009, 11, 2647.

[13] Zhang, W.-S.; Kuang, C.-X.; Su, C.-H. Chem. Reag. 2009, 31, 261 (in Chinese).

(张文生, 匡春香, 苏长会, 化学试剂, 2009, 31, 261.)

[14] Pawluć, P.; Hreczycho, G.; Szudkowska, J.; Kubicki, M.; Marciniec, B. Org. Lett. 2009, 11, 3390.

[15] Yu, M.; Zhang, G. Z.; Zhang, L. M. Tetrahedron 2009, 65, 1846.

[16] Ye, L.; Zhang, L. Org. Lett. 2009, 11, 3646.

[17] Shen, X. Q.; Hyde, A. M.; Buchwald, S. L. J. Am. Chem. Soc. 2010, 132, 14076.

[18] Pan, J.; Wang, X.; Zhang, Y.; Buchwald, S. L. Org. Lett. 2011, 13, 4974.

[19] Imazaki, Y.; Shirakawa, E.; Ueno, R.; Hayashi, T. J. Am. Chem. Soc. 2012, 134, 14760.

[20] Zhang, W. S.; Kuang, C. X.; Yang, Q. Chin. J. Chem. 2009, 27, 1727.

[21] Kuang, C. X.; Senboku, H.; Tokuda, M. Tetrahedron Lett. 2001, 42, 3893.

[22] Bull, J. A.; Mousseau, J. J.; Charette, A. B. Org. Lett. 2008, 10, 5485.

[23] Li, W.; Li, J. C.; Wan, Z. K.; Wu, J. J.; Massefski, W. Org. Lett. 2007, 9, 4607.

[24] Wang, Y.; Lam, H. W. J. Org. Chem. 2009, 74, 1353.

[25] Yin, J. D.; Gallis, C. E.; Chisholm, J. D. J. Org. Chem. 2007, 72, 7054.

[26] Kutsumura, N.; Niwa, K.; Saito, T. Org. Lett. 2010, 12, 3316. 